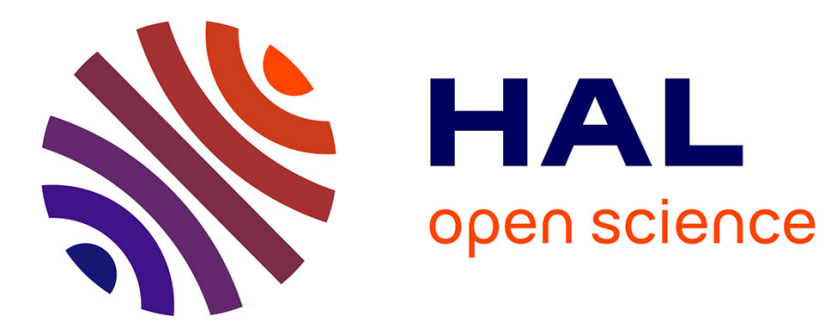

\title{
Electrostatic method to estimate the mechanical properties of suspended membranes applied to nickel-coated graphene oxide
}

Nawres Sridi, Bérengère Lebental, Joel Azevedo, Jean Christophe Gabriel, Anne Ghis

\section{To cite this version:}

Nawres Sridi, Bérengère Lebental, Joel Azevedo, Jean Christophe Gabriel, Anne Ghis. Electrostatic method to estimate the mechanical properties of suspended membranes applied to nickelcoated graphene oxide. Applied Physics Letters, 2013, 103 (05-1907), 5p. 10.1063/1.4817301 . hal00860866v2

\section{HAL Id: hal-00860866 \\ https://hal.science/hal-00860866v2}

Submitted on 29 Jun 2017

HAL is a multi-disciplinary open access archive for the deposit and dissemination of scientific research documents, whether they are published or not. The documents may come from teaching and research institutions in France or abroad, or from public or private research centers.
L'archive ouverte pluridisciplinaire HAL, est destinée au dépôt et à la diffusion de documents scientifiques de niveau recherche, publiés ou non, émanant des établissements d'enseignement et de recherche français ou étrangers, des laboratoires publics ou privés. 


\title{
Electrostatic method to estimate the mechanical properties of suspended membranes applied to nickel-coated graphene oxide
}

\author{
Nawres Sridi, ${ }^{1}$ Berengere Lebental, ${ }^{2,3}$ Joel Azevedo, ${ }^{4}$ Jean Christophe P. Gabriel, ${ }^{5}$ \\ and Anne Ghis ${ }^{1, a)}$ \\ ${ }^{1}$ CEA, LETI, Minatec, 17 rue des Martyrs, 38054 Grenoble Cedex 9, France \\ ${ }^{2}$ Université Paris-Est, Ifsttar, 58 Boulevard Lefebvre, 75732 Paris Cedex, France \\ ${ }^{3}$ LPICM Ecole Polytechnique, Route de Saclay, 91128 Palaiseau Cedex, France \\ ${ }^{4}$ CEA Saclay, IRAMIS, Service de Physique de l'Etat Condensé, 91191 Gif sur Yvette, France \\ ${ }^{5}$ CEA, Physical Science Division, 17 rue des Martyrs, 38054 Grenoble Cedex 9, France
}

(Received 17 April 2013; accepted 14 July 2013; published online 31 July 2013)

\begin{abstract}
We propose a method to estimate the bending rigidity and Young's modulus of thin conducting suspended membranes based on measuring the deflection of the membranes submitted to an electrostatic force. Our electrostatic method appears easier to implement and more reliable than AFM-based localized force-displacement measurements to estimate the bending rigidity and Young's modulus of slightly inhomogeneous materials. We apply the method on suspended graphene oxide (GO) sheets coated with a $5 \mathrm{~nm}$ thick Ni layer, providing a demonstration of electrostatic actuation for GO sheets. For a $7.7 \mathrm{~nm}$ thick membrane, a Young modulus of $360 \mathrm{GPa}$ is found. (C) 2013 AIP Publishing LLC. [http://dx.doi.org/10.1063/1.4817301]
\end{abstract}

Graphene, like other two dimensional materials, ${ }^{1}$ offers a wide range of $\mathrm{sp}^{2}$-specific physical and chemical properties $^{2}$ which has motivated its integration into a variety of devices, such as electromechanical resonators ${ }^{3}$ and actuators ${ }^{4}$ field effect transistors, ${ }^{5}$ or electrochemical sensors. ${ }^{6}$ In the field of NanoElectromechanical Systems (NEMS), graphene is invaluable because of its high stiffness, its high surface over volume ratio, and its high sensitivity to environmental changes. ${ }^{7,8}$ A variety of graphenes resulting from the chemical exfoliation of graphite or graphite oxide, ${ }^{9}$ graphene oxide (GO), often under reduced form (RGO), ${ }^{10}$ has attracted attention for device fabrication, as it is easier than pristine graphene to fabricate, process, and functionalize, ${ }^{11}$ while it has similar mechanical properties. ${ }^{12}$ However, no examples of GO or RGO based NEMS devices are reported in the literature, because GO and RGO conductivities remain too low for electrostatic actuation. Here, we fabricate an electrostatically actuated suspended GO sheets. Sufficient conductivity is obtained by coating the GO sheet with a thin nickel layer.

While the nickel coating is used to ensure the overall conductivity of the material, its Young's modulus is quite low. ${ }^{13}$ The stiffness required for electromechanical applications is expected from the GO sheets. However, once processed and integrated, the mechanical properties of graphene and GO/RGO sheets are known to widely differ from theoretical expectations and from bulk material properties, ${ }^{12,14}$ so that the overall mechanical properties of the suspended membranes cannot be anticipated.

It is a hurdle for further use of the material into NEMS devices, as any difference in the mechanical properties, especially in the Young's modulus $E$, may affect device operation $^{15}$ (resonance frequency, quality factor, bandwidth, amplitude of vibrations, operating voltage, etc.) through a change in the bending rigidity $D$, defined for a plate-like

a)anne.ghis@cea.fr material as $\mathrm{D}=\mathrm{Et}^{3} / 12\left(1-\nu^{2}\right)$ with $\nu$ the Poisson's ratio and $t$ the thickness. ${ }^{16}$

The bending rigidity is often estimated from AFM based localized force-displacement measurements, ${ }^{17,18}$ resonance frequency measurements, ${ }^{19}$ or bulge test method. ${ }^{20}$ More recently, the snap-through voltage of convex-buckled membranes has been used to estimate $D .{ }^{21}$ Alternately, the bending rigidity may be calculated from the Young's modulus derived by nanoindentation, ${ }^{22}$ Raman spectroscopy, ${ }^{23}$ or $a b$ initio calculations. ${ }^{24}$

Here, we propose a method to estimate the bending rigidity and Young's modulus of suspended metal-coated GO sheets, and more generally of slightly inhomogeneous, thin, conducting membranes, following their integration into an electromechanical system. Our method relies on measuring by AFM the membrane deflection under electrostatic actuation. This approach is compared to results from AFM localized force-displacement experiments.

Devices are fabricated on $\mathrm{Si}(\mathrm{n}++) / \mathrm{SiO}_{2}(200 \mathrm{~nm})$ substrates with $\mathrm{Cr} / \mathrm{Au}(5 / 100 \mathrm{~nm})$ top-side electrodes. The electrodes are shaped into pairs of $1 \mathrm{~mm}$-long, $150 \mu \mathrm{m}$-wide rectangles spaced by a gap ranging from $1.4 \mu \mathrm{m}$ to $5 \mu \mathrm{m}$. To serve as a conducting under-layer for the graphene oxide sheets, we first deposit a $5 \mathrm{~nm}$ thin nickel film by electron gun evaporation (Plassys MEB 550S). Nickel is used for its compatibility with the rest of the fabrication process (especially availability of deposition method and selectivity of wet etching) as well as for its good compatibility with graphene-based material as a graphene catalyst. ${ }^{25}$ High purity $(>90 \%)$ graphite oxide is exfoliated in ultra-pure water for $90 \mathrm{~min}$ to obtain $\mathrm{GO}$ suspension at a concentration of $4 \mathrm{mg} / \mathrm{ml}$. A film of overlapping GO flakes is formed on the Ni layer by three successive depositions using the bubble deposition method. The deposition process is detailed elsewhere. ${ }^{26}$ The average flake diameter is $3 \mu \mathrm{m}$. The average thickness of a single GO flake is $1.5 \mathrm{~nm} \pm 0.3 \mathrm{~nm} .{ }^{26}$ The thickness of the GO film depends on the folding of the flakes 
and the number of superposed flakes. The GO sheets lying over the Ni layer are patterned into $20 \mu \mathrm{m}$ by $250 \mu \mathrm{m}$ stripes using optical lithography on AZ 1512 HS photo-resist followed by oxygen plasma etching. ${ }^{3}$ Aqueous hydrochloric acid at $12 \mathrm{~mol} / \mathrm{l}$ is then used at room temperature to etch away the nickel not covered by GO and photoresist. Finally, the underlying $\mathrm{SiO}_{2}$ layer is etched away to suspend the thin stripes. To prevent stiction during rinsing and drying, ${ }^{27} \mathrm{HF}$ is used in vapor phase at room temperature for $2 \mathrm{~min}$ (Ref. 28) (see Fig. 1).

Membranes are deflected downward electrostatically by applying a DC bias voltage $V$ between the suspended membrane and the highly doped Si substrate. ${ }^{17}$ The AFM tip scans lines in tapping mode to acquire the vertical profile of the membranes along the suspended span. The suspended profiles are recorded for both null and non-null voltage $V$, and the vertical deflection is derived point by point as the difference between the two profiles. The voltage changes are applied very slowly so that the system remains in quasistatic regime.

Measurements are performed on several NiGO stripes under different bias voltages. Figure 2 presents the deflection with respect with position and the maximum deflection (at the middle of the suspended span) with respect to voltage.

An analytical model relying on Kirchoff-Love plate theory ${ }^{29}$ is used to fit the experimental data and deduce the bending rigidity. The rectangular plate is assumed to be elastic, homogeneous, isotropic, and thin. The thin plate hypothesis is valid as the ratio of span $(1.4-1.8 \mu \mathrm{m})$ to thickness (lower than $15 \mathrm{~nm}$ ) is larger than 20 . We study the pure bending regime ${ }^{16}$ (deflections smaller than thickness) in static regime.

Let us denote $l$ the span of the plate, $y$ (resp. $z$ ) the spatial coordinate in the direction parallel (resp. perpendicular) to the trench axis, and $w(y, z)$ the vertical deflection (see Fig. 1). The system can be assumed translation-invariant along the $y$ axis, so that $w(y, z)=w(z)$.

The equation governing the deflection $w$ under an electrostatic force per unit area $Q(w(z))$ is

$$
D \frac{d^{4} w(z)}{d z^{4}}=Q(w(z))=\frac{\epsilon V^{2}}{2\left(d_{0}-w(z)\right)^{2}},
$$

with $D$ the bending rigidity, $\epsilon$ the permittivity of the gap material (here air), and $d_{0}$ the initial distance between membrane and substrate.
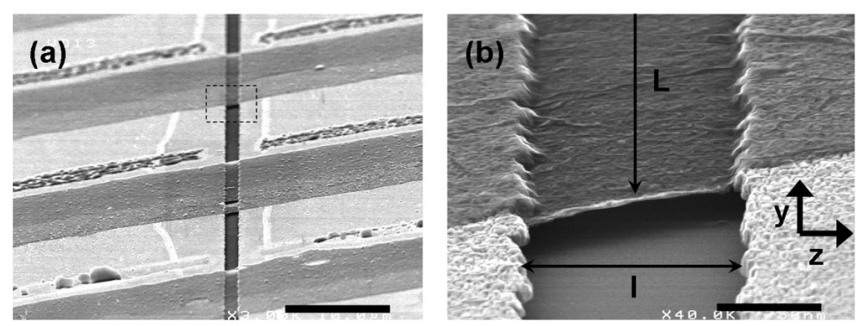

FIG. 1. SEM images of nickel-coated graphene oxide stripes suspended over a $200 \mathrm{~nm}$ deep, $1.4 \mu \mathrm{m}$ wide trench. (a) Stripes are $20 \mu \mathrm{m}$ wide and $250 \mu \mathrm{m}$ long and are spaced by $30 \mu \mathrm{m}$. Scale bar: $10 \mu \mathrm{m}$. (b) Zoomed image of the edge of a suspended stripe. Scale bar: $750 \mathrm{~nm}$.
With $w(z) \ll d_{0}$ (small displacement regime), $Q(w(z))$ can be linearized as

$$
Q_{\operatorname{lin}}(w(z)) \simeq \frac{\epsilon V^{2}}{2 d_{0}^{2}}\left(1+\frac{2 w(z)}{d_{0}}\right)
$$

Solving the fourth-order linear differential Eq. (1) under the linearized force 2 , we find

$$
w(z)=-\frac{d_{0}}{2}+C_{1} \cos \left(\alpha\left(z-\frac{l}{2}\right)+C_{2} \cosh \left(\alpha\left(z-\frac{l}{2}\right),\right.\right.
$$

with $\alpha=\left(\epsilon \mathrm{V}^{2} / \mathrm{Dd}_{0}^{3}\right)^{1 / 4}$. The coefficients $\mathrm{C}_{1,2}$ are calculated using the boundary conditions of a rectangular plate with two opposite clamped edges and two opposite free edges.

The experimental deflection-versus-position profiles are fitted by the least square method using Eq. (3) with the bending rigidity $D$ as fitting parameter (see Figure 2). Results are summarized in Table I.

In order to validate the electrostatic approach, the bending rigidity of the membranes is calculated from AFM forcedisplacement measurements. The suspended membranes are loaded by lowering the AFM tip. The vertical force applied by the tip and the membrane displacement at the tip contact point are simultaneously acquired, yielding one forcedisplacement $F(\delta)$ curve for each contact point. The portion of this curve corresponding to small displacements (with respect to thickness) is linear (the whole curve is generally well fitted by a third order polynom), and its slope yields the spring constant of the membrane at the contact point. We acquire the force-displacement curves $F(\delta)$ on a regular $32 \times 32$ grid taken over a $3 \mu \mathrm{m} \times 3 \mu \mathrm{m}$ area containing the suspended zone and both its edges. After calibration, linear portions of $F(\delta)$ curves are fitted with a $k_{l} \delta$ model, $k_{l}$ being the local spring constant.

The bending rigidity is deduced from the spring constant at the center of the membrane using linear analytical models. For suspended 1D materials such as nanotubes ${ }^{30,31}$ and nanowires,${ }^{17}$ the linear clamped beam model (Euler-Bernouilli beam theory) is used to derive the relationship between the spring constant at the middle of the beam $k_{m}$ and the Young's modulus $E: k_{m}=192 \frac{E t^{3} w}{12 l^{\beta}}$, where $t, w$, and $l$ are, respectively, the thickness, the width, and the length of the beam. For plate geometries, the relationship is significantly different and depends strongly on the boundary conditions. For circular plates of radius $r$ with clamped edges, the analytical model $k_{m}=16 \pi D \frac{1}{r^{2}}$ derived in Ref. 16 is used. ${ }^{32,33}$ For rectangular plates with two opposite edges clamped and two opposite edges free, one of the most common experimental configuration for graphene-like material, the analytical derivation of the deflection is highly involved: no author has ever provided an analytical, exact, or approximate, relationship between the spring constant at the center of the plate and the bending rigidity. The beam relationship is commonly used, ${ }^{18,23,34}$ but is expected to yield significantly underestimated values for the bending rigidity and the Young's modulus.

Here we use finite elements to derive an approximate analytic expression for the spring constant at the center of the plate. The suspended membrane is modeled as a 

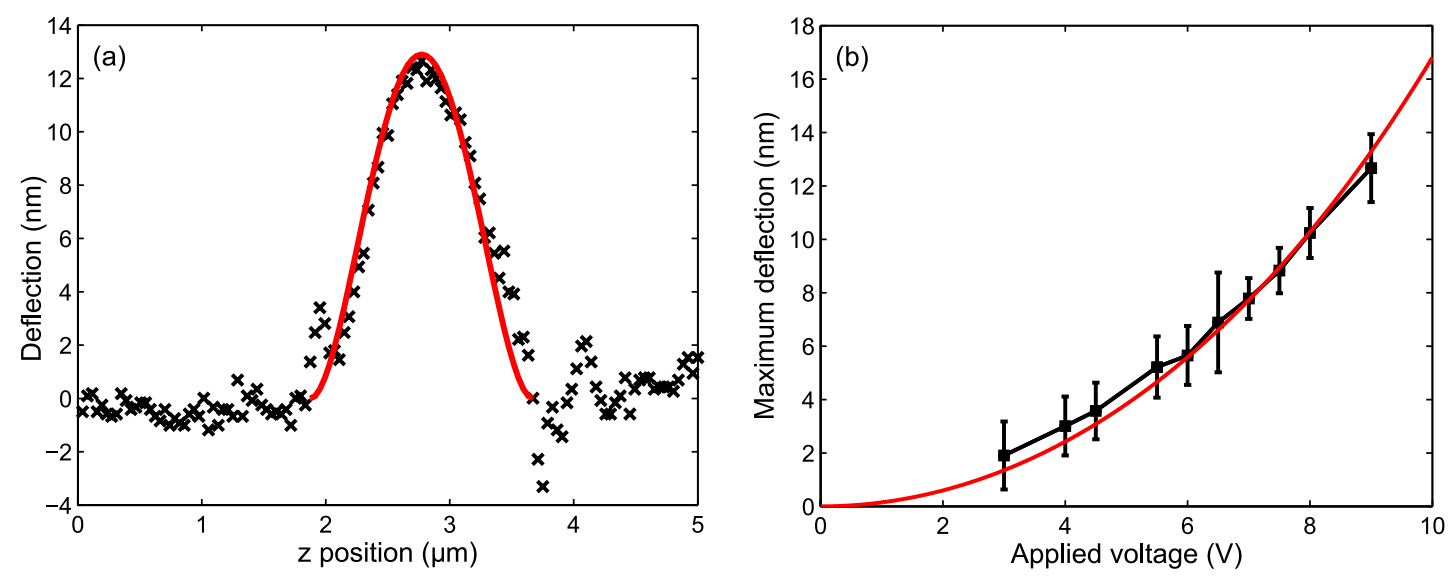

FIG. 2. (a) Deflection of a $1.8 \mu \mathrm{m}$ wide and $8.1 \mathrm{~nm}$ thick membrane at DC bias of $9 \mathrm{~V}$. Experimental measures (in black crosses) are fitted (red lines) by the linear model (3). (b) Maximum deflection (at the center of the membrane) with respect to DC bias voltage (in black squares). Measurements are fitted (red line) using (3).

rectangular, elastic, homogeneous, isotropic, thin (thickness t) plate clamped along its long edges (size $L$ ) and free along its short edges (size $l$ ). The force is applied at the center and is modeled as a point load because the radius of the AFM tip (around $10 \mathrm{~nm}$ ) is much smaller than the short edge $l$ and the characteristic size of finite element meshes suitable to such geometries. Pre-stress is neglected.

The relationship between force $F$ and deflection at the center $\delta$ is derived as follows: according to the linear plate model, $\mathrm{F} / \delta$ is proportional to $\mathrm{E} /\left(1-\nu^{2}\right)$. We verify numerically that $\delta$ is independent from $L$ as soon as $\mathrm{L}>1$. Then, with $\mathrm{L}=20 \mu \mathrm{m}$, we calculate the plate deflection $\delta$ for 16 different $(t, l)$ pairs, with $t$ ranging from $10 \mathrm{~nm}$ to $30 \mathrm{~nm}$ and $l$ ranging from $0.5 \mu \mathrm{m}$ to $1.8 \mu \mathrm{m}$. Using these 16 values for the deflections, we establish with better than $2 \%$ accuracy (fit by least square method) the relationship

$$
k_{m}=137 \frac{E}{12\left(1-\nu^{2}\right)} \frac{t^{3}}{l^{2}}=137 \frac{D}{l^{2}} .
$$

Using this analytical relationship (4) between spring constant and bending rigidity, we derive the bending rigidity of the NiGO stripes as summarized in Table II.

The values obtained for the bending rigidity of the $1.4 \mu \mathrm{m}$ wide membrane can be divided into three groups (as identified in Table II). We postulate that these groups correspond to three different thicknesses $\left(t_{1}, t_{1}+1.5, t_{1}+3\right) \mathrm{nm}$ corresponding to an increasing number of overlapping GO flakes. ${ }^{26}$ The best fit for the thickness is $t_{1}=7.7 \pm 1.6 \mathrm{~nm}$, corresponding to $\mathrm{E}=360 \mathrm{GPa}$ for the Young's modulus. Table II and the Figure 3 summarize the different thicknesses derived using this value for $\mathrm{E}$. Using the classical relationship between Young's modulus and thickness for composite

TABLE I. Bending rigidity of the NiGO stripes deduced from the AFMmeasured displacements under electrostatic actuation.

\begin{tabular}{lccc}
\hline \hline Label & Gap $(\mu \mathrm{m})$ & $\mathrm{D}\left(10^{-14} \mathrm{~N} . \mathrm{m}\right)$ & Fit error $(\%)$ \\
\hline $\mathrm{a}$ & 1.4 & 9.3 & 26 \\
$\mathrm{~b}$ & & 12 & 24 \\
$\mathrm{c}$ & 1.8 & 2.1 & 15 \\
\hline \hline
\end{tabular}

materials undergoing bending $\left(\mathrm{Et}_{1}=\mathrm{t}_{N i} \mathrm{E}_{N i}+\mathrm{t}_{G O} \mathrm{E}_{G O}\right)^{35}$ and using literature value for thin $\mathrm{Ni}$ films $\left(\mathrm{E}_{N i}=240 \mathrm{GPa}\right.$ for $20 \mathrm{~nm}$ thick Ni (Ref. 36)), we deduce $\mathrm{E}_{G O}=580 \pm 120 \mathrm{GPa}$. This value is about twice larger than typical experimental values for GO. Three factors can explain this elevated value: an underestimation of the Ni Young's modulus is likely, as the Young's modulus varies with the film thickness. ${ }^{37}$ Additionally, the GO here has been transferred by the bubble deposition method, which is expected to induce very little structural damage in the material. ${ }^{38}$ Finally, pre-stress has been neglected in our calculation. If NiGO membranes are pre-stressed, an overestimation of the bending rigidity and the Young's modulus is induced.

The values of bending rigidity obtained with the electrostatic approach for devices a, b, and c would correspond to $14.5 \mathrm{~nm}, 15.8 \mathrm{~nm}$, and $8.8 \mathrm{~nm}$ thick membranes, respectively. This is in agreement with the measured thickness using AFM in contact mode ( $8.1 \mathrm{~nm}$ for device $\mathrm{c}$ in Table I).

Contrary to the classical AFM-based method which relies on localized measurements highly sensitive to heterogeneity and defects, our electrostatic approach accounts for the global mechanical behavior of the membrane. Hence, the influence of technological artifacts and measurement noise is lowered. Furthermore, the electrostatic method is easier to implement since it does not require prior (Sader modelbased) derivation of the AFM cantilever spring constant. ${ }^{39}$ Derivation of the intrinsic mechanical parameters from the measurements is easier too, as the models for homogeneous forces are much more easily derived analytically for any geometric configuration. Admittedly the electrostatic method

TABLE II. Bending rigidity and thickness of the NiGO stripes.

\begin{tabular}{cccccc}
\hline \hline No & $l(\mu \mathrm{m})$ & $D\left(10^{-14} \mathrm{~N} . \mathrm{m}\right)$ & $\operatorname{Std}(\%)$ & Group & $t(\mathrm{~nm})$ \\
\hline 1 & 1.2 & 24 & $\mathbf{4}$ & 7.7 \\
2 & 1.4 & 23 & $\mathbf{4}$ & \\
3 & & 1.6 & 14 & $\mathbf{4}$ & \\
4 & \multirow{2}{*}{1.4} & 2.3 & 16 & $\mathbf{0}$ & 9.2 \\
5 & & 2.5 & 13 & $\mathbf{0}$ & \\
6 & & 3.6 & 10 & $\mathbf{4}$ & 10.7 \\
7 & 3.9 & 16 & $\mathbf{4}$ & \\
\hline \hline
\end{tabular}




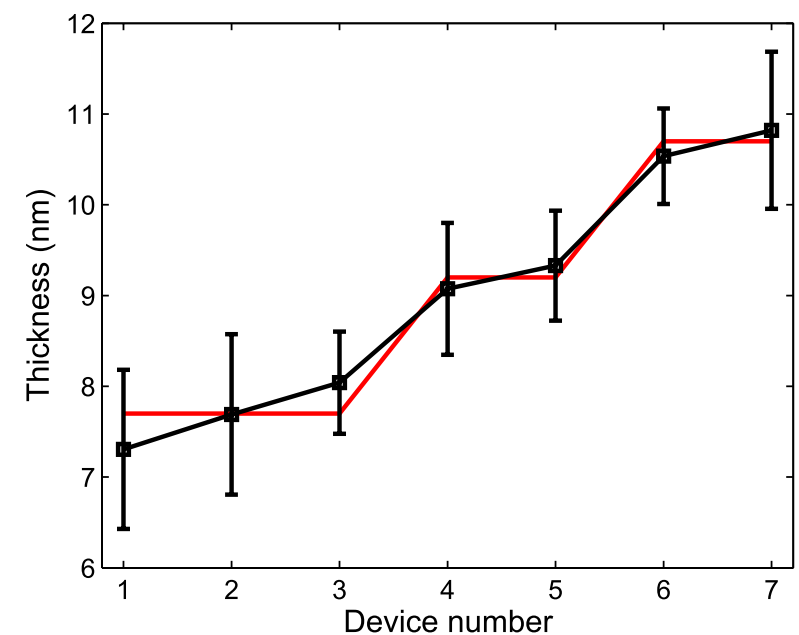

FIG. 3. In black squares, the estimated thickness of each measured device with error bars. In red line, theoretical thickness corresponding to $\left(t_{1}, t_{1}+1.5, t_{1}+3\right) \mathrm{nm}$.

requires the prior electrical connection of the electrodes and implies that the membrane is entirely suspended in order to avoid short circuits, but these requirements are similar to what any transducer device should satisfy and are thus not reducing the impact of our method for electromechanical systems.

In this paper, we propose a method to determine the bending rigidity of suspended membranes which relies on measuring by tapping-mode AFM the deflection of the membrane under electrostatic loading and on interpreting the data using an analytical plate model.

We apply this method to the electrostatically actuated suspended GO membranes. Sufficient conductivity of the membrane material is obtained using a thin nickel coating. Results are found to agree with bending rigidity values determined using the reference method relying on localized AFM force-displacement curves.

Compared to this reference method, our electrostatic method appears more suitable to assess the properties of slightly inhomogeneous or defect-free materials. It is significantly easier to implement, and the results are more straightforward to analyze in terms of bending rigidity. This technique is not limited to NiGO films but can be applied to any other kind of suspended conducting materials.

${ }^{1}$ K. Teii, R. Yamao, and S. Matsumoto, J. Appl. Phys. 106, 113706 (2009).

${ }^{2}$ K. Novoselov, D. Jiang, F. Schedin, T. Booth, V. Khotkevich, S. Morozov, and A. Geim, Proc. Natl. Acad. Sci. U.S.A. 102, 10451 (2005).

${ }^{3}$ D. Garcia-Sanchez, A. M. van der Zande, A. S. Paulo, B. Lassagne, P. L. McEuen, and A. Bachtold, Nano Lett. 8, 1399 (2008).
${ }^{4}$ J. Liang, L. Huang, N. Li, Y. Huang, Y. Wu, S. Fang, J. Oh, M. Kozlov, Y. Ma, F. Li, R. Baughman, and Y. Chen, ACS Nano 6, 4508 (2012).

${ }^{5}$ B. Hahnlein, B. Handel, J. Pezoldt, H. Tapfer, R. Granzner, and F. Schwierz, Appl. Phys. Lett. 101, 093504 (2012).

${ }^{6}$ M. Pumera, A. Ambrosi, A. Bonanni, E. L. K. Chng, and H. L. Poh, TrAC, Trends Anal. Chem. 29, 954 (2010).

${ }^{7}$ Y. Shao, J. Wang, H. Wu, J. Liu, I. Aksay, and Y. Lin, Electroanalysis 22, 1027 (2010).

${ }^{8}$ P. Han, H. Wang, Z. Liu, X. Chen, W. Ma, J. Yao, Y. Zhu, and G. Cui, Carbon 49, 693 (2011).

${ }^{9}$ H. Yang, Y. Hernandez, A. Schlierf, A. Felten, A. Eckmann, S. Johal, P. Louette, J.-J. Pireaux, X. Feng, K. Mullen, V. Palermo, and C. D. Casiraghi, Carbon 53, 357 (2013).

${ }^{10}$ S. Pei and H.-M. Cheng, Carbon 50, 3210 (2012).

${ }^{11}$ D. R. Dreyer, S. Park, C. W. Bielawski, and R. S. Ruoff, Chem. Soc. Rev. 39, 228 (2010).

${ }^{12}$ J. W. Suk, R. D. Piner, J. An, and R. S. Ruoff, ACS Nano 4, 6557 (2010).

${ }^{13}$ S.-H. Kim and J. G. Boyd, Meas. Sci. Technol. 17, 2343 (2006).

${ }^{14}$ C. Gomez-Navarro, M. Burghard, and K. Kern, Nano Lett. 8, 2045 (2008).

${ }^{15}$ T. Wah, J. Acoust. Soc. Am. 34, 275 (1962).

${ }^{16} \mathrm{~S}$. Timoshenko and S. Woinowsky-Krieger, Theory of Plates and Shells, edited by N. Y. Engineering Societies Monographs (McGraw-Hill, 1959).

${ }^{17}$ A. San Paulo, J. Bokor, R. T. Howe, R. He, P. Yang, D. Gao, C. Carraro, and R. Maboudian, Appl. Phys. Lett. 87, 053111 (2005).

${ }^{18}$ I. W. Frank, D. M. Tanenbaum, A. M. van der Zande, and P. L. McEuen, J. Vac. Sci. Technol. B 25, 2558 (2007).

${ }^{19}$ B. Ilic, S. Krylov, and H. Craighead, J. Appl. Phys. 108, 044317 (2010).

${ }^{20}$ S. Maruthoor, A. Ajayakumar, T. Fuchs, O. Jakovlev, H. Reinecke, and J. Wilde, J. Microelectromech. Syst. 22, 140 (2013).

${ }^{21}$ N. Lindahl, D. Midtvedt, J. Svensson, O. A. Nerushev, N. Lindvall, A. Isacsson, and E. E. B. Campbell, Nano Lett. 12, 3526 (2012).

${ }^{22}$ A. Richter, R. Ries, R. Smith, M. Henkel, and B. Wolf, Diamond Relat. Mater. 9, 170 (2000).

${ }^{23}$ J.-U. Lee, D. Yoon, and H. Cheong, Nano Lett. 12, 4444 (2012).

${ }^{24}$ Y. Wei, B. Wang, J. Wu, R. Yang, and M. L. Dunn, Nano Lett. 13, 26 (2013).

${ }^{25}$ L. Baraton, Z. He, C. Lee, J.-L. Maurice, C. Cojocaru, A.-F. GourguesLorenzon, Y. Lee, and D. Pribat, Nanotechnology 22, 085601 (2011).

${ }^{26}$ J. Azevedo, C. Costa-Coquelard, P. Jegou, T. Yu, and J.-J. Benattar, J. Phys. Chem. C 115, 14678 (2011).

${ }^{27}$ H. Kim, C. Kim, M. Yu, H.-S. Kim, and R. H. Blick, J. Appl. Phys. 108, 074307 (2010).

${ }^{28}$ K. R. Williams, K. Gupta, and M. Wasilik, J. Microelectromech. Syst. 12, 761 (2003).

${ }^{29}$ A. E. H. Love, "On the small free vibrations and deformations of elastic shells," in Philosophical Transactions of the Royal Society (London), edited by Transactions of the Royal Society of London (1888), pp. 491-549.

${ }^{30}$ S. W. Lee, B. Kim, D. S. Lee, H. J. Lee, J. G. Park, S. J. Ahn, E. E. B. Campbell, and Y. W. Park, Nanotechnology 17, 992 (2006).

${ }^{31}$ L. Niu, X. Chen, S. Allen, and S. J. B. Tendler, Langmuir 23, 7443 (2007).

${ }^{32}$ A. Castellanos-Gomez, M. Poot, G. Steele, H. van der Zant, N. Agrait, and G. Rubio-Bollinger, Nanoscale Res. Lett. 7, 233 (2012).

${ }^{33}$ S. Bertolazzi, J. Brivio, and A. Kis, ACS Nano 5, 9703 (2011).

${ }^{34}$ P. Li, Z. You, G. Haugstad, and T. Cui, Appl. Phys. Lett. 98, 253105 (2011).

${ }^{35}$ E. Date, J. Phys. D 3, 778-782 (1970).

${ }^{36}$ C.-T. Wang, S.-R. Jian, J.-C. Jang, Y.-S. Lai, and P.-F. Yang, Appl. Surf. Sci. 255, 3240-3250 (2008).

${ }^{37}$ A. I. Fedorchenko, A.-B. Wang, and H. H. Cheng, Appl. Phys. Lett. 94, 152111 (2009).

${ }^{38}$ G. Andreatta, Y. J. Wang, F. K. Lee, A. Polidori, P. Tong, B. Pucci, and J.-J. Benattar, Langmuir 24, 6072-6078 (2008).

${ }^{39}$ J. E. Sader, J. W. M. Chon, and P. Mulvaney, Rev. Sci. Instrum. 70, 3967 (1999). 